\title{
Autoevaluación y gamificación en las prácticas de Anatomía Humana en el Grado de Terapia Ocupacional
}

\section{Julia Dugnol Menéndez ${ }^{a}$, María Isabel Fernández Méndez ${ }^{b}$, Juliana Pérez Suárez ${ }^{\mathfrak{y}}$ María Luisa Ruiz Fernández ${ }^{\mathrm{d}}$}

aLicenciada en Biología por la Universidad de Oviedo. Profesora de prácticas de Anatomía Humana

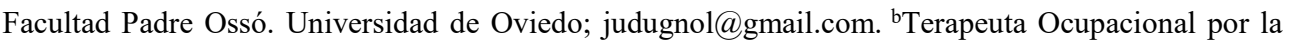
Universidad de Oviedo. Profesora de Terapia Ocupacional Facultad Padre Osó. Universidad de Oviedo; isabelf@facultadpadreosso.es. 'Doctora en Biología por la Universidad de Oviedo. Profesora de Biología y Fisiología Humana del Grado de Terapia Ocupacional. Vicedecana de Calidad Facultad Padre Ossó. Universidad de Oviedo; julianap@facultadpadreosso.es. ${ }^{\mathrm{d} D o c t o r a}$ en Medicina y Cirugía por la Universidad de Oviedo. Médico Especialista en Medicina de la Educación Física y el Deporte. Profesora de Anatomía Humana y Geriatría y Patologías Osteoarticulares. Grado Terapia Ocupacional. Facultad Padre Ossó. Universidad de Oviedo; luisa@facultadpadreosso.es.

\begin{abstract}
The purpose of this study is to create a learning tool that facilitates teamwork and improves student learning capacity and autonomy. Two workbooks of human anatomy for occupational therapists were created, since according to the current scientific literature none exist. The students covered during the laboratory sessions. Once students were considered by staff to have the necessary knowledge, the students carried out an online test. If they were able to achieve a score of 5, they were allowed access to the workbook solutions. All the material is hosted on the virtual platform of the faculty (Moodle). The subject was also evaluated with two gamified tests. As indicators of students' performance, grades obtained during the course, the practical examination based on the gamification and the assessment of the students were evaluated together.
\end{abstract}

Keywords: workbooks, Anatomy, Occupational Therapy, innovation, autonomy, virtual platform, gammification

\section{Resumen}

El propósito de este estudio es crear una herramienta de aprendizaje que facilite el trabajo en equipo y mejore la capacidad de aprendizaje y la autonomía de los estudiantes. Se elaboraron dos cuadernos prácticos de Anatomía Humana centrados en aparato locomotor y sistema nervioso para Terapia Ocupacional, dado que carece de texto específico en la literatura científica actual. El alumnado los completó durante las prácticas de la asignatura. Una vez consideraban haber adquiridos los conocimientos, realizaban un cuestionario online. Si superaban la calificación de 5, podrían acceder al solucionario del cuaderno. Todo el material se encontraba alojado en la plataforma virtual de la facultad (moodle). La asignatura fue evaluada, además, con dos pruebas gamificadas. Como indicadores, se 
valoraron las calificaciones obtenidas, el examen práctico basado en la gamificación y la satisfacción del alumnado.

Palabras clave: cuadernos prácticos, Anatomía, Terapia Ocupacional, innovación, autonomía, plataforma virtuay gamificación.

\section{Introducción}

Los conocimientos anatomo-funcionales del cuerpo humano resultan esenciales en la formación del alumnado perteneciente a la rama de conocimiento de Ciencias de la Salud y, en concreto, en el caso de los estudiantes del Grado de Terapia Ocupacional. Con ellos el alumno consigue la base para poder adquirir competencias y habilidades en otras materias del Grado, de manera que la Anatomía se considera básica para estos estudiantes.

Uno de los objetivos fundamentales del proceso de Convergencia Europea fue promover el cambio metodológico en la Enseñanza Superior. Además de los cambios relativos a la organización de las enseñanzas - Nuevo Catálogo de Títulos Universitarios - y en el sistema de cómputo de la actividad académica - Créditos ECTS - la filosofía de la Convergencia pretendió impulsar un proceso de renovación de la metodología que habitualmente se venía utilizando en la enseñanza universitaria. Frente al paradigma tradicional que centra el eje de la enseñanza sobre la tarea del profesor, el Espacio Europeo de Educación Superior (EEES) promueve una teoría basada en el supuesto de que sólo se logra un aprendizaje eficaz cuando es el propio alumnado el que asume la responsabilidad en la organización y desarrollo de su trabajo académico. Aceptar este principio supone enfocar necesariamente los procesos de enseñanza desde una perspectiva distinta a la actual, ya que el centro de la actividad pasa del profesor al estudiante. En el momento actual nos corresponde, por tanto, a los docentes avanzar en el desarrollo de nuevos modelos de aprendizaje más efectivos (uso estratégico de nuevos recursos educativos puestos a su disposición), que logren un mayor nivel de aprendizaje en el alumnado.

\section{Objetivos}

\subsection{Objetivos generales}

El desarrollo de nuevos métodos de aprendizaje incorporando las Tecnologías de la Información y Comunicación en la oferta formativa.

\subsection{Objetivos específicos}

Implementar una metodología práctica que facilite el aprendizaje y la autonomía del alumnado en la asignatura de Anatomía Humana.

\section{Desarrollo de la innovación}

\subsection{Plan de trabajo desarrollado}


$\mathrm{Al}$ inicio del curso se alojó en la plataforma virtual (moodle) el material didáctico necesario para las prácticas de Anatomía Humana, poniendo a su disposición material bibliográfico y presentaciones en Power Point acorde al temario establecido en la guía docente. Se entregaron, siempre en la plataforma virtual, 13 capítulos de Aparato Locomotor y un capítulo final de repaso, divididos de la siguiente forma:

1. Generalidades, 2. Cavidad craneal, 3. Macizo facial, 4. Columna vertebral, 5. Caja torácica, 6. Miembro superior, 7. Miembro inferior, 8. Artrología, 9. Músculos del dorso, 10. Músculos de la pared abdominal, 11. Músculos del miembro superior, 12. Músculos del miembro inferior, 13. Topografía, 14. Cuestionario de repaso (Fig. 1).
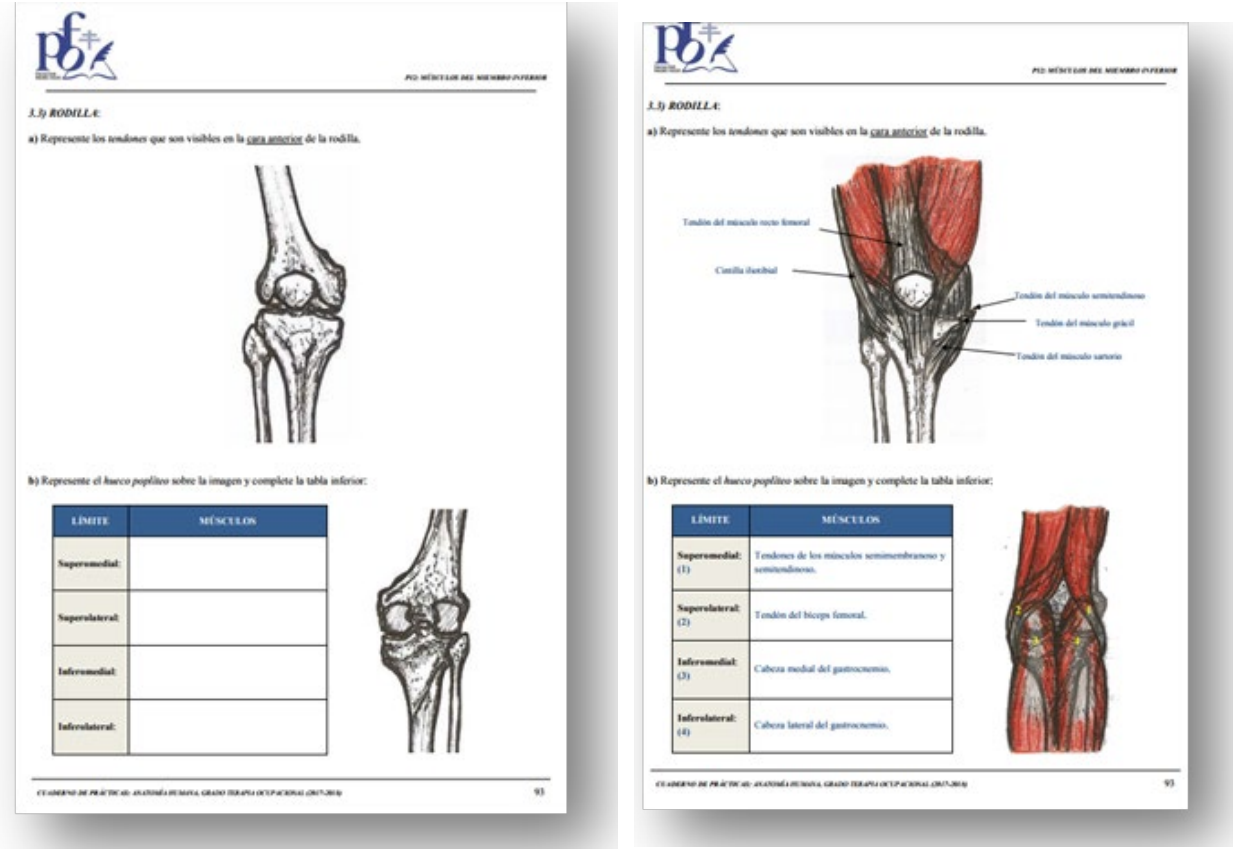

Fig. 1: Ejemplo de hoja del cuaderno de actividad y del solucionario de Aparato Locomotor

Del cuaderno de Sistema Nervioso se alojaron siete capítulos y uno más de repaso, divididos en: 1. Generalidades, 2. Cerebro, 3. Cerebelo, 4. Tronco del encéfalo, 5. Médula espinal, 6. Pares craneales y órganos de los sentidos, 7. Sistema nervioso autónomo, 8. Cuestionario de repaso (Fig. 2). 

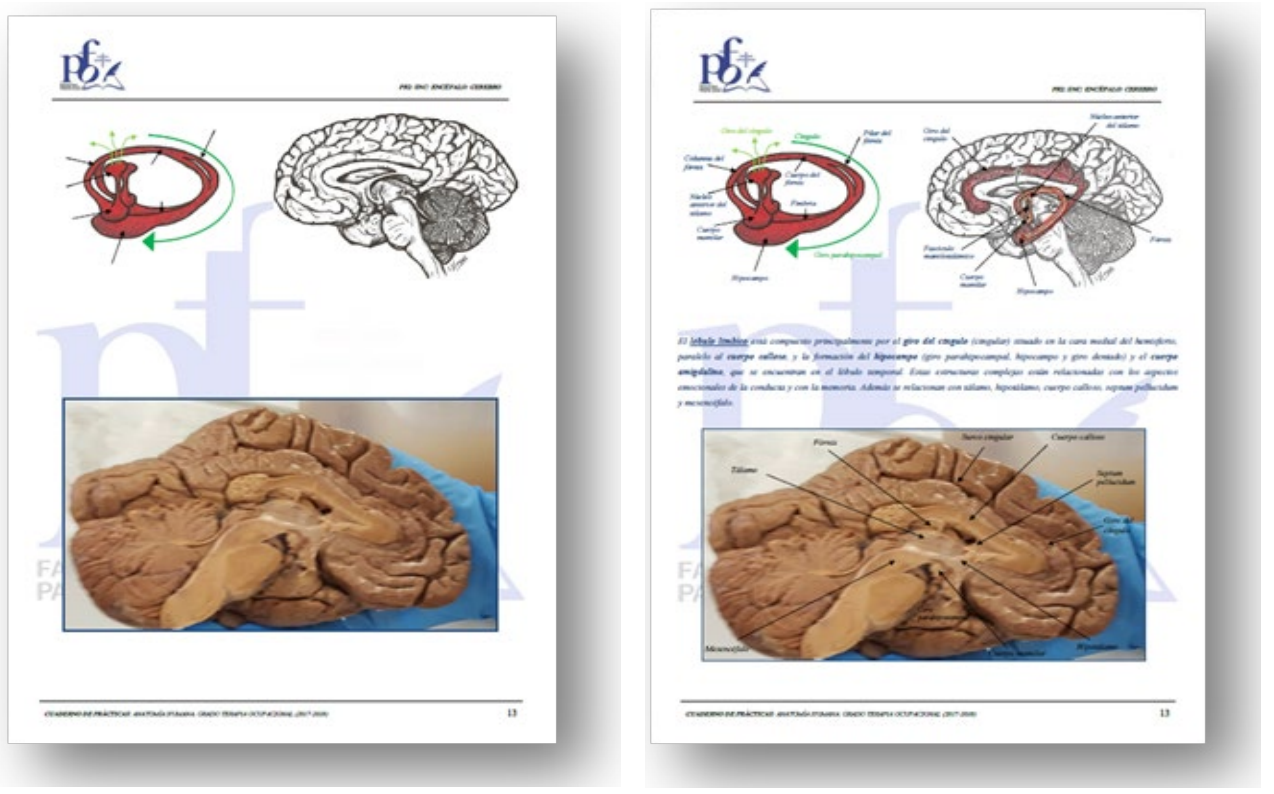

Fig. 2: Ejemplo de hoja del cuaderno de actividad y del solucionario del Sistema Nervioso

Así mismo, disponían del cuestionario adaptado a cada epígrafe (Fig. 3).

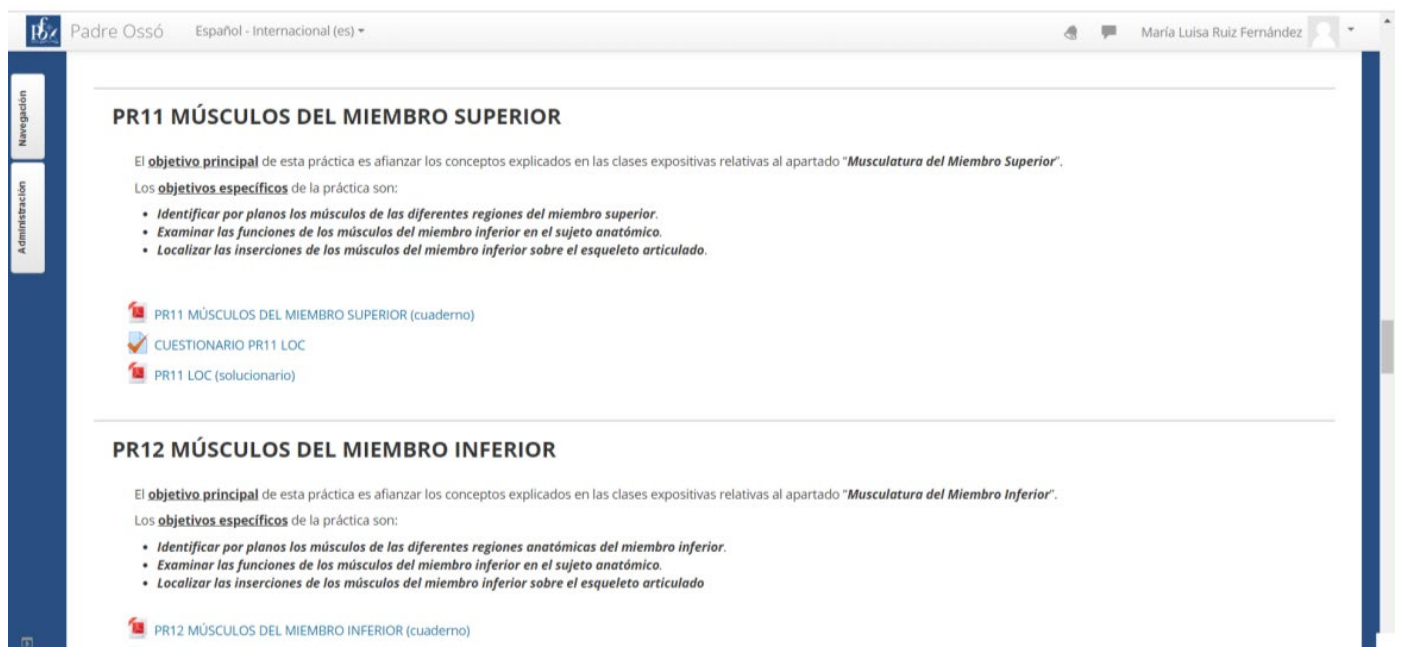

Fig. 3: Vista en moodle del cuaderno, cuestionario y solucionario de cada capitulohttps://moodle.facultadpadreosso.es/course/view.php?id=266

\subsection{Descripción de la metodología}

Cada capítulo se correspondía con una sesión práctica en sala dedisección de la Facultad de Medicina de la Universidad de Oviedo. Durante la misma, los estudiantes trabajaban en grupo tutorizados por dos profesores, mientras se resolvían las dudas planteadas en los cuadernos con maquetas y preparaciones de cadáver (Fig. 4). 


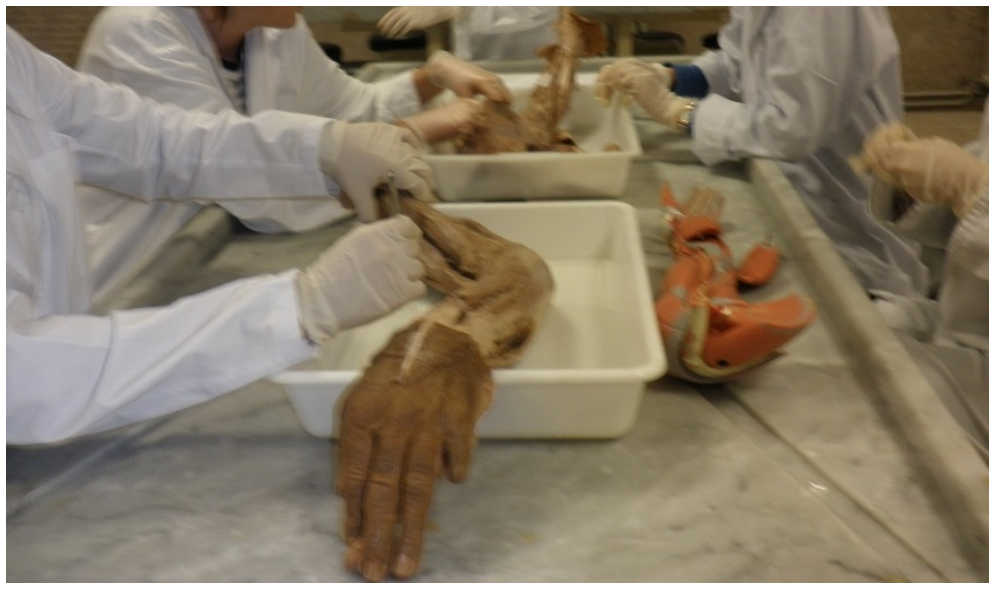

Fig. 4: Estudiantes en sala de disección trabajando Aparato Locomotor

Los cuestionarios se configuraron para tener fecha límite de resolución. La calificación de los mismo, así como el tiempo empleado y el número de intentos (disponían de tres intentos y cuatro minutos) necesitados para obtener una puntuación igual o superior a 5, son visibles para el docente en todo momento. Una vez superada la fecha límite, se abrían los solucionarios para permitir a aquellos alumnos que no hubieran superado la calificación disponer también del solucionario de los cuadernos (Fig. 5).

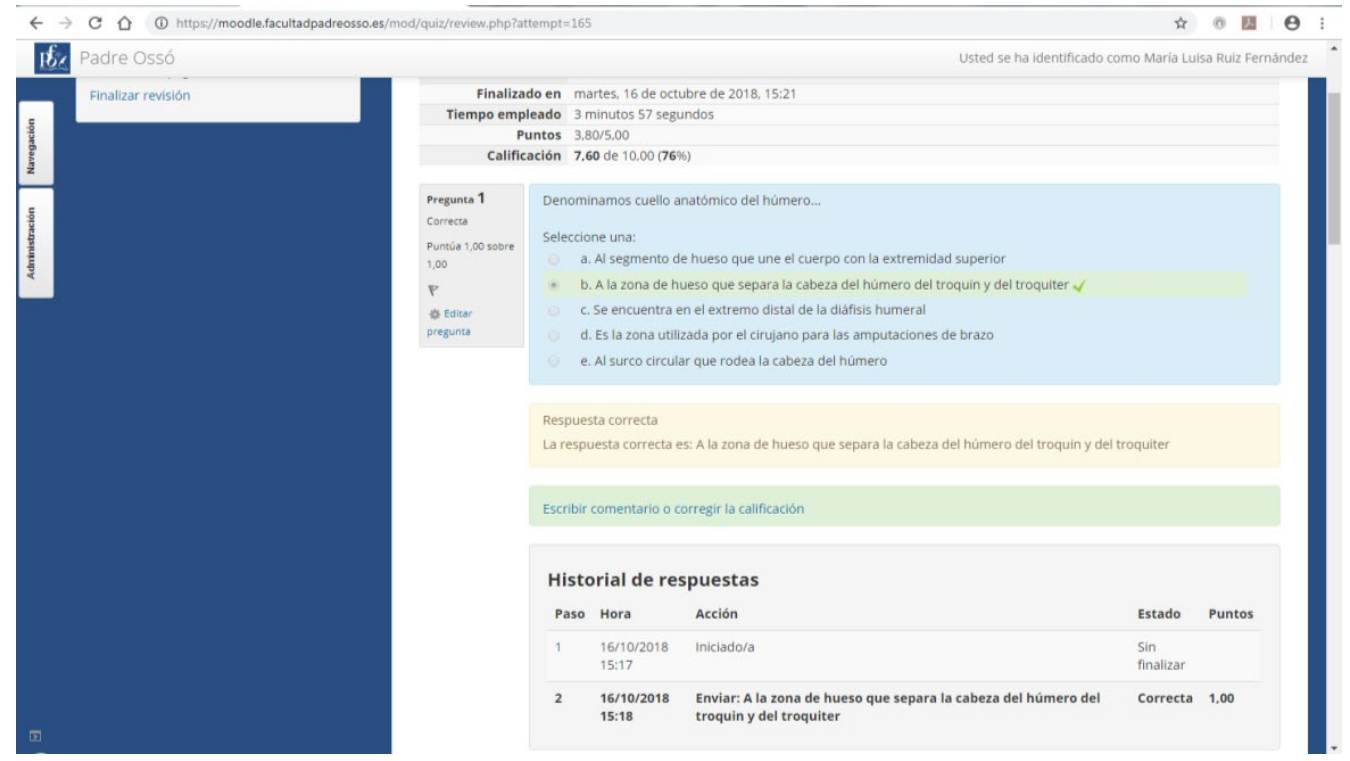

Fig. 5: Ejemplo de cuestionario

El diseño del examen práctico se basó en la gamificación. Debido a que los estudiantes realizan las prácticas en grupos, se propuso un diseño grupal de las pruebas. Una de ellas tuvo lugar al finalizar el temario del Aparato Locomotor y consistió en utilizar su propio cuerpo para representar las diferentes inserciones musculares y demostrar la funcionalidad de las mismas (Figs. 6 y 7 ). 


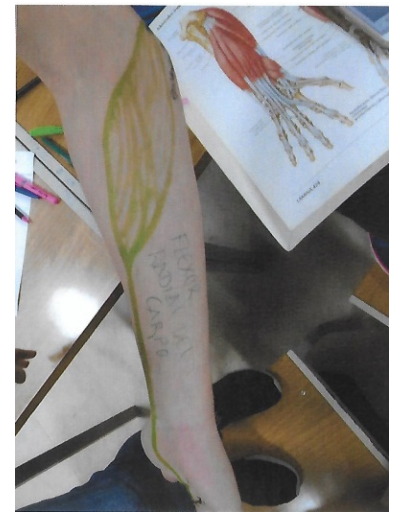

Fig. 6: Estudiante realizando la representación muscular en su antebrazo

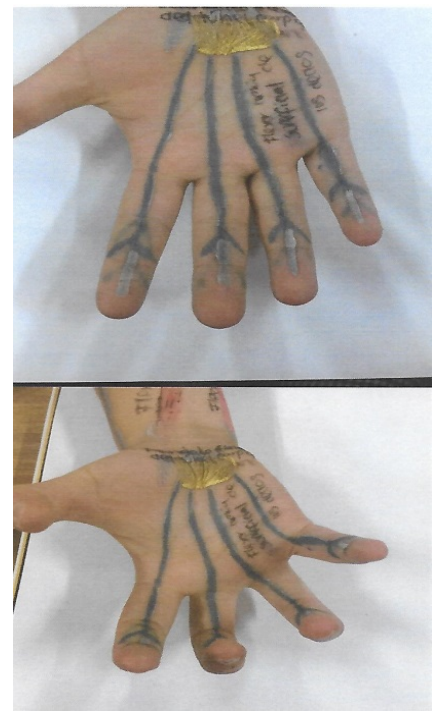

Fig. 7: Representación de la funcionalidad del músculo pintado sobre su piel

La segunda prueba, basada en la gamificación (Figs. 8 y 9), se realizó al finalizar el Sistema Nervioso y consistió en una prueba similar a una "gincana": 1) Cada grupo tenía una pregunta que debía contestar y de la cuál obtenía un número. 2) Ese número dirigía al grupo hacia una segunda parte, donde había unas mesas numeradas con maquetas o preparaciones de cadáver sobre ellas y en las que debían identificar determinadas estructuras. 3) Por último, tenían que localizar las mismas estructuradas en cortes de macizo craneofacial situados en una tercera mesa. 


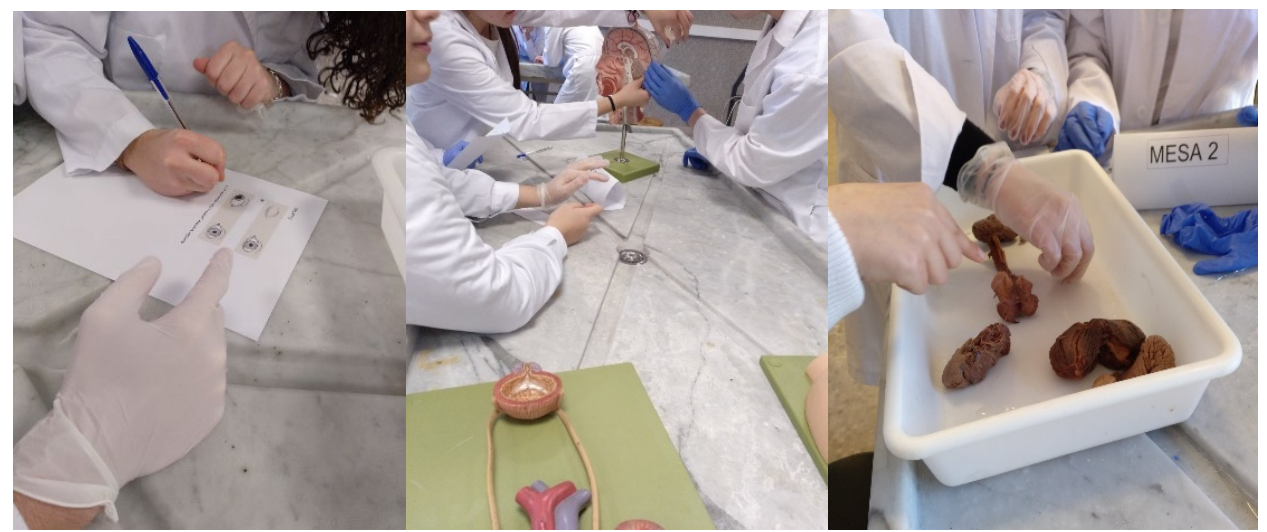

Fig. 8: Estudiantes realizando la gamificación de la primera y segunda prueba

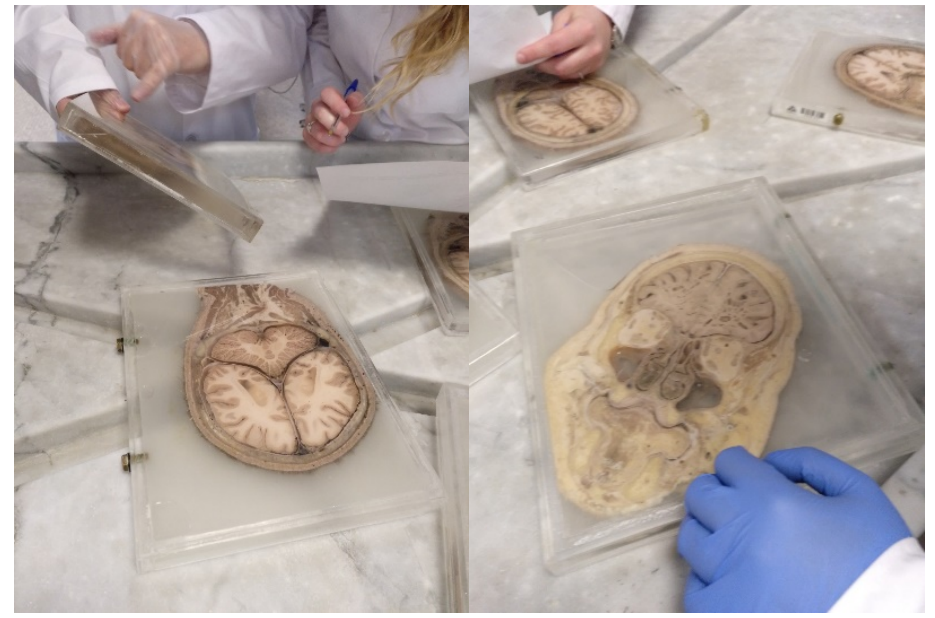

Fig. 9: Estudiantes localizando estructuras anatómicas en cortes de preparación de cadáver

\section{Resultados alcanzados}

\subsection{Valoración de indicadores académicos}

Como indicadores académicos hemos valorado las calificaciones obtenidas tanto en el examen parcial, primer indicador, como en la convocatoria ordinaria al finalizar el curso, segundo indicador (Tabla 1). Así mismo, valoramos la media obtenida por el alumnado en los cuestionarios online.

Tabla1. Indicadores planteados para valoración del estudio

\begin{tabular}{|l|l|l|l|}
\hline $\mathbf{N}^{\mathbf{0}}$ & \multicolumn{1}{|c|}{ Indicador } & \multicolumn{1}{c|}{ Modo de evaluación } & \multicolumn{1}{c|}{ Rangos fijados y obtenidos } \\
\hline 1 & $\begin{array}{l}\text { Número de alumnos/as } \\
\text { que superen la puntuación } \\
\text { de } 6\end{array}$ & $\begin{array}{l}\text { Examen tipo test de } 5 \text { repuestas; solo } \\
\text { una válida con 0,20 de penalización } \\
\text { por respuesta errónea. }\end{array}$ & $\begin{array}{l}\text { Entre } 0.0 \%-40 \%=>\text { Bajo } \\
\text { Entre } 40 \%-80 \%=>\text { Aceptable } \\
\text { Por encima del } 80 \%=>\text { Bueno } \\
\text { Obtenido 46\% }=>\text { ACEPTABLE }\end{array}$ \\
\hline
\end{tabular}




\begin{tabular}{|c|c|c|c|}
\hline $\mathbf{N}^{\circ}$ & Indicador & Modo de evaluación & Rangos fijados y obtenidos \\
\hline 2 & $\begin{array}{lcr}\text { Número de } & \text { aprobados } \\
2018 / 2019 & \text { frente } & \text { a los } \\
\text { últimos } & 5 & \text { cursos } \\
\text { académicos. } & & \\
\end{array}$ & $\begin{array}{l}\text { Se tomarácomoindicador las notas de } \\
\text { la convoctoria ordinaria que figuran en } \\
\text { acta }\end{array}$ & $\begin{array}{l}\text { Finales: } \\
\text { Entre } 0.0 \%-50 \%=>\text { Bajo } \\
\text { Entre } 50 \%-100 \%=>\text { Aceptable } \\
\text { Obtenido } 64 \%=>\text { ACEPTABLE }\end{array}$ \\
\hline 3 & $\begin{array}{lr}\text { Número de } & \text { alumnos/as } \\
\text { que } & \text { completen } \\
\text { correctamente } & \text { los } \\
\text { cuadernos } & \end{array}$ & $\begin{array}{l}\text { Corrección individualizada de cada } \\
\text { cuaderno }\end{array}$ & $\begin{array}{l}\text { Entre } 0.0 \%-40 \%=>\text { Bajo } \\
\text { Entre } 40 \%-80 \%=>\text { Aceptable } \\
\text { Por encima del } 80 \%=>\text { Bueno } \\
\text { Obtenido BUENO }\end{array}$ \\
\hline
\end{tabular}

En las calificaciones del primer parcial (Fig. 10) hemos mejorado los resultados obtenidos hasta ahora; según nuestros objetivos iniciales es ACEPTABLE. Del total del alumnado presentado al examen de Aparato Locomotor superaron la nota de corte, calificación de 6 o superior, el $46 \%$. Son porcentajes superiores a los obtenidos en los cinco últimos años que eran del $20 \%$ del alumnado presentado. Además, hemos constatado que el $58 \%$ del alumnado que eliminó materia lo hizo con calificaciones superiores al 7; este porcentaje es similar al curso 2017-2018, donde ya se utilizaron cuadernos y han sido muy superiores a los obtenidos en años anteriores.

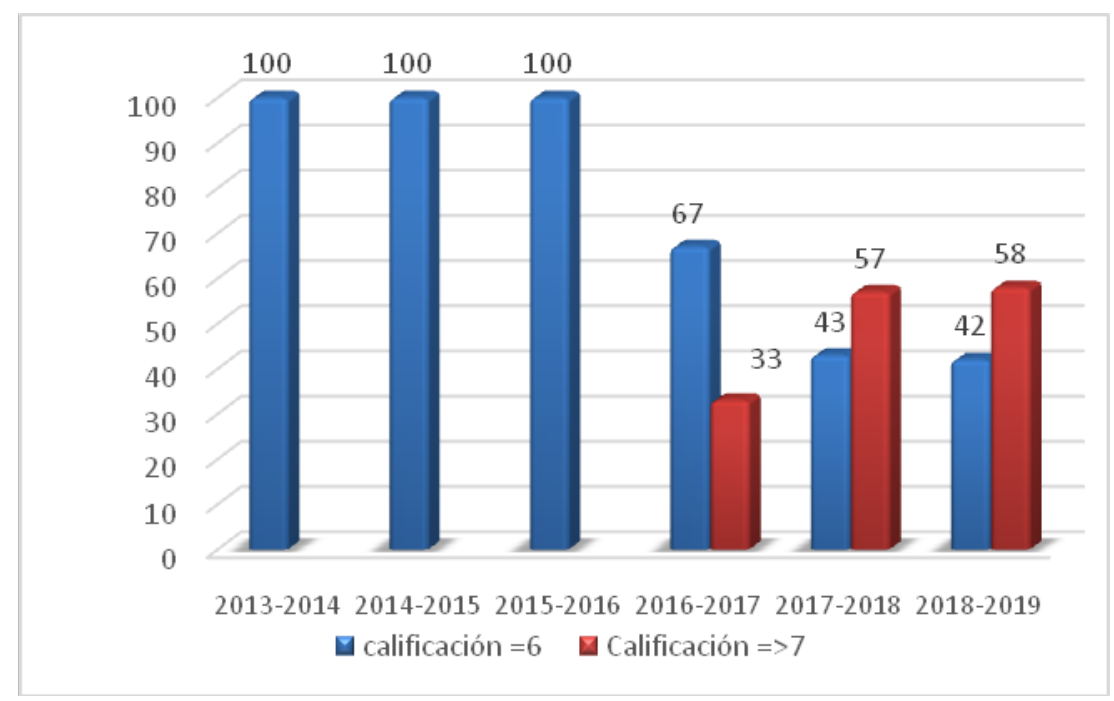

Fig. 10: Gráfico se representa la calificación que obtuvo el alumnado que superó el primer parcial, en porcentajes. Se representala comparativa de los cinco últimos años

El número de estudiantes que han superado la materia en convocatoria ordinaria fue del $64 \%$, porcentaje similar a curso previos. Hemos observado una mejora en las calificaciones, con un porcentaje significativamente superior en las calificaciones de: notable, sobresaliente y matrícula de honor (Fig. 11). 


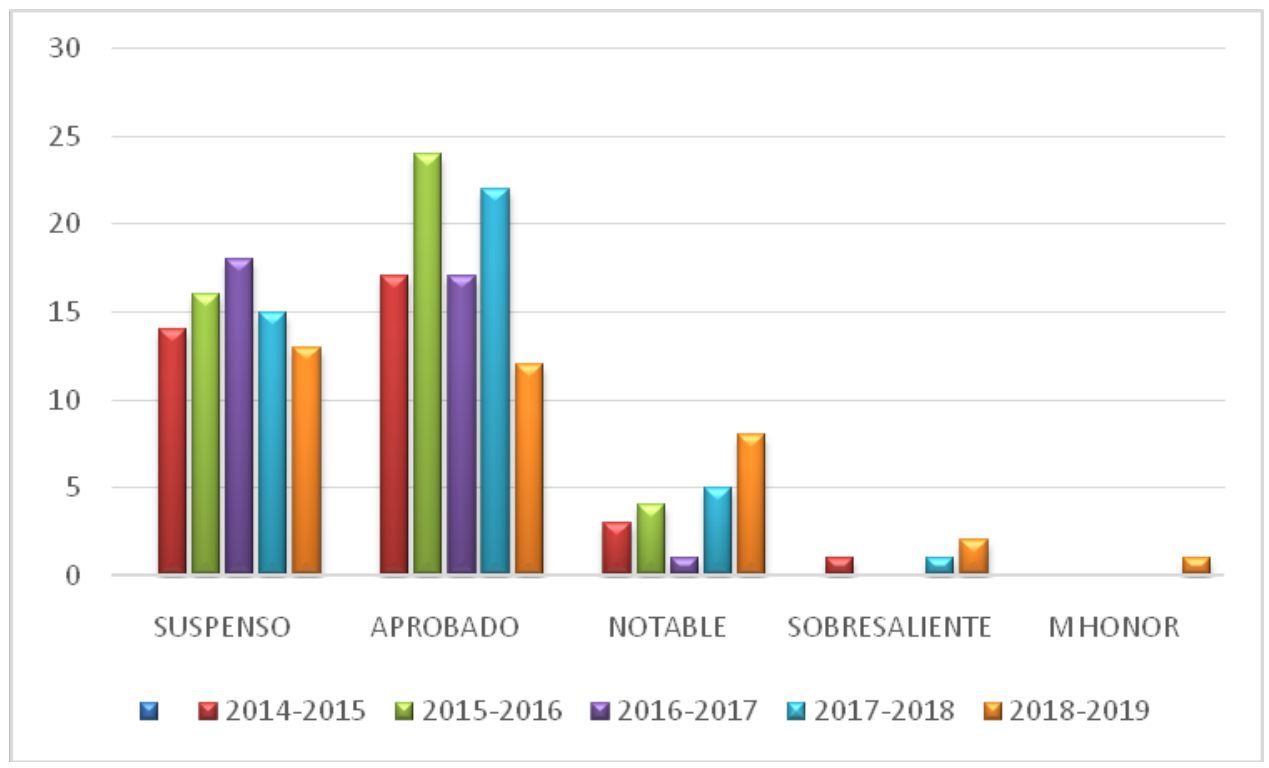

Fig. 11: Gráficos que muestran la representación de la calificación del alumnado en convocatoria ordinaria. Se representa la comparativa con años anteriores

El número de estudiantes que superó en el tiempo establecido los cuestionarios y con la puntuación necesaria para acceder al solucionario ha sido casi del $90 \%$, con lo que ese parámetro tiene la categoría de BUENO. La media obtenida es de 6,42 para el cuaderno de Aparato Locomotor y 5,98 para el cuaderno de Sistema Nervioso.

El alumnado ha mostrado una clara preferencia por este método. Se ha conseguido una implicación elevada en la resolución de los cuadernos, puesto que tan solo el $10 \%$ del alumnado los dejó sin realizar. Además, se ha conseguido obtener unas calificaciones superiores a cursos académicos anteriores y hemos dotado a nuestros estudiantes de una herramienta de estudio y de consulta específica para el Grado de Terapia Ocupacional.

En cuanto a las pruebas gamificadas, la calificación obtenidaen las dos prácticas gamificadas ha sido superior a la práctica no gamificada (presentación grupal en PowerPoint) (Fig. 12). 


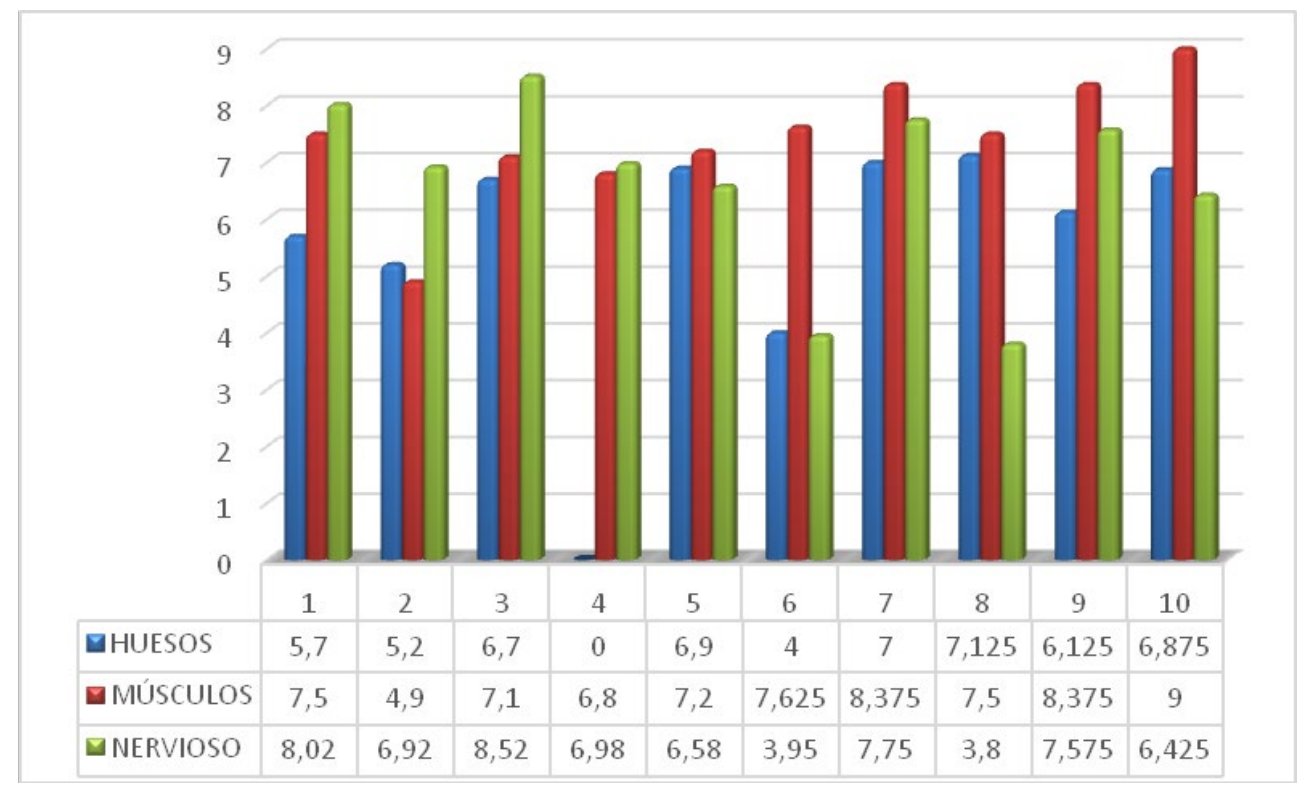

Fig. 12: Gráficos que muestran la representación de la calificación del alumnado en las prácticas realizadas alo largo del curso actual. Se representa por grupos

\subsection{Valoración del grado de satisfacción}

Incluimos un cuestionario de satisfacción, "grado de satisfacción del alumnado", tanto sobre el uso del cuaderno y cuestionario (Tabla 2) como de la gamificación para la evaluación de las prácticas (Tabla 3). Dichos cuestionarios contienen tres grupos de ítems: evaluación de los cuadernos/gamificación, nivel de dificultad y satisfacción general. Creemos necesaria la opinión del alumnado para conseguir su implicación en el aprendizaje.

Tabla 2. Encuesta de satisfacción del alumnado puntuación media obtenida

\begin{tabular}{|c|c|c|c|c|c|}
\hline \multicolumn{6}{|l|}{$\begin{array}{l}\text { EVALUACIÓN DE LOS CUADERNOS: } 5 \text { estoy muy de acuerdo; } 1 \text { estoy en total } \\
\text { desacuerdo }\end{array}$} \\
\hline & 1 & 2 & 3 & 4 & 5 \\
\hline Considera que la secuenciación de la actividad práctica es la adecuada & & & & 4,3 & \\
\hline La división de capítulos es adecuada & & & & 4,2 & \\
\hline La división de capítulos facilita el aprendizaje & & & & 4,2 & \\
\hline El contenido del cuaderno completa el aprendizaje de las clases expositivas & & & 3,6 & & \\
\hline La sincronización temporal de clase expositiva, prácticas y cuaderno es apropiada & & & 3,8 & & \\
\hline Los enunciados son comprensibles y están bien redactados & & & & 4 & \\
\hline Los cuadernos han sido utilizados de manera efectiva & & & & 4,4 & \\
\hline NIVEL DE DIFICULTAD: 5 estoy muy de acuerdo; 1 estoy en total desacuerdo & & & & & \\
\hline & 1 & 2 & 3 & 4 & 5 \\
\hline
\end{tabular}


La realización delos cuadernos me ha resultado didáctico
La realización delos cuadernos me ha resultado amena
La realización delos cuadernos me ha resultado difícil

SATISFACCIÓN GENERAL: 5 estoy muy de acuerdo; 1 estoy en total desacuerdo

Al terminar los cuadernos siento que han aumentado mis conocimientos de la materia

Los cuadernos han aumentado mi interés en la materia

L os cuadernos me han afianzado los conocimientos teóricos

En general las actividades prácticas han cubierto mis expectativas

Recomiendo seguir usando cuadernos

¿Qué no quitaría nunca de los cuadernos?

¿Qué quitaría de los cuadernos?

Una vez finalizados los cuadernos y las pruebas gamificadas, se realizaron las encuestas de satisfacción. Sobre los cuadernos, más del $80 \%$ considera que son efectivos, que facilitan el aprendizaje, que tienen una correcta sincronización con las clases y las prácticas. El 85\% lo considera didáctico y, la mitad de ellos, ameno; solo un 19\% lo considera difícil. El mismo porcentaje, el 19\%, recomienda no seguir usándolos frente al $81 \%$ que dice querer usarlos siempre. Mejora los porcentajes del anterior curso académico. Sobre la gamificación, el $73 \%$ seguiría con esta metodología ya que facilita el aprendizaje de forma amena y didáctica.

Tabla 3. Encuesta de satisfacción del alumnado con la gamificación. Porcentajes de respuestas

\section{ENCUESTA DE SATISFACCIÓN}

Marcar con una X la casilla correspondiente "siendo 1 totalmente en desacuerdo y 5 totalmente de acuerdo"

\section{EVALUACIÓN DE LA GAMIFICACIÓN}

1 Considero que se ajusta a los contenidos teóricos

2 El contenido de los mismos es claro y fácilmente entendible

3 Facilita el aprendizaje

\begin{tabular}{|r|r|r|r|r|}
\hline 1 & 2 & 3 & 4 & 5 \\
\hline & & & 21 & $\mathbf{7 8}$ \\
\hline & & 9,7 & 24,2 & $\mathbf{6 0 , 9}$ \\
\hline & & 14,6 & 14 & $\mathbf{7 0 , 7}$ \\
\hline 4,8 & $\mathbf{3 2}$ & $\mathbf{3 1 , 7}$ & 24,3 & 7,3 \\
\hline
\end{tabular}

\section{VALORACIÓN DE LOS JUEGOS}

1 Me resultó difícil
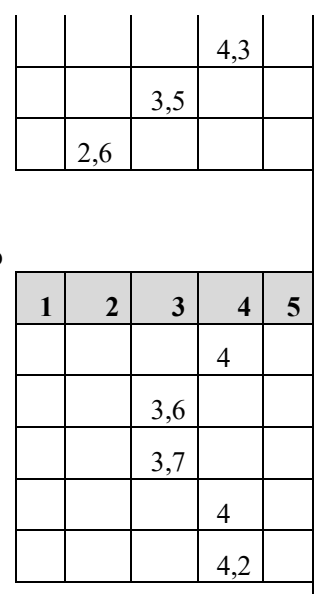
2 Me resultó ameno

3 Me resulto didáctico

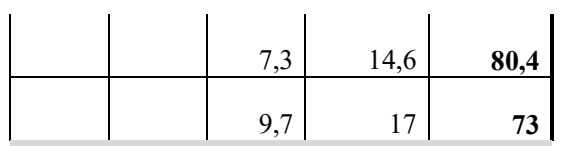

\section{GRADO DE SATISFACCIÓN GENERAL}

$1 \mathrm{Al}$ terminarlos ha aumentado mi conocimiento de las materias

2 Ha aumentado mi interés en las materias

3 Me ayudó a afianzar los conocimientos teóricos

4 Cumplió mis expectativas

5 Recomiendo continuar esta metodología

6 Preferiría volver al sistema anterior sin gamificación

\begin{tabular}{|r|r|r|r|r|} 
& 12 & 22 & $\mathbf{4 6}$ & 19,5 \\
\hline & 4,8 & 19 & 29 & $\mathbf{4 6 , 3}$ \\
\hline & 9,7 & 12 & 36 & $\mathbf{4 1 , 4}$ \\
\hline 2,4 & & 7,3 & 22 & $\mathbf{7 0 , 7}$ \\
\hline 4,8 & 2,4 & 4,8 & 17 & $\mathbf{7 3}$ \\
\hline $\mathbf{4 6}$ & 24 & 4,8 & 12 & 4,8 \\
\hline
\end{tabular}

OBSERVACIONES

\section{Conclusiones}

La primera conclusión es que tenemos una herramienta específica para el estudio de Anatomía Humana en Terapia Ocupacional que fomenta el trabajo autónomo y grupal del alumnado, objetivado por el alto número de estudiantes que completó el trabajo. Fomentamos el trabajo autónomo del alumnado haciendolos conscientes de la adquisición de conocimientos necesarios para solucionar los cuestionarios, y fomentamos el trabajo grupal basándonos en la resolución de problemas. La herramienta facilitada es valorada muy positivamente por las y los estudiantes como facilitadora del aprendizaje, objetivado por la mejoraen las calificaciones obtenidas.

La segunda conclusión es que la gamificación como herramienta evaluadora es valorada de forma muy positiva por el alumnado. Creemos que estimula la participación del alumnado en la resolución de problemas, disminuyendo el estrés frente a técnicas convencionales e individuales de evaluación, logrando mejores resultados académicos.

Estamos potenciando el uso de nuevas tecnologías, permitiendo de esta forma al alumnado el acceso al material desde cualquier dispositivo electrónico. El alto grado de implicación del alumnado tanto en la resolución de los cuadernos como en la gamificación y la mejora en las calificaciones, nos hacen valorar positivamente el método, si bien creemos necesario realizar modificaciones dirigidas a implementar esta metodología en materias transversales del Grado.

\section{Referencias}

ARGÜELLES PABON, D.C. (2010). Estrategias para promover procesos de aprendizaje autónomo. Bogotá DC: Universidad EAN. 
ERRINGTON, E. (2001). "The influence of teacher beliefs on flexible learning innovation in traditional university settings" en Lockwood, F., Gooley, A. Innovation in open and distance learning. Successful development of online and web-based learning. Londres: Kogan Page Limited, p. 27-37.

DE MIGUEL DiAZ, F.M. (2005). "Cambio de paradigma metodológico en la educación superior. Exigencias que conlleva" en Cuadernos de integración europea, vol. 2, p. 16-27. https://cdeuv.es/documents/2005-CIE-02.pdf.

PARMAR, S.K., RATHINAM B.A. (2011). "Introduction of vertical integration and case-based learning in anatomy for undergraduate physical therapy and occupational therapy students" en Anatomical Sciences Education, 4(3), p. 170-173.

RASCH, P.J., BURKE, R.K. (1989). Kinesiology and applied anatomy. Estados Unidos: Lea \& Febiger.

RUE, J. (2009). El aprendizaje autónomo en la Educación Superior. Madrid: Narcea.

SCHOFIELD, K.A. (2014). "Anatomy in occupational therapy program curriculum: Practitioners' perspectives" en Anatomical Sciences Education, 7(2), p. 97-106.

SUGAND, K., ABRAHAMS P., KHURANA, A. (2010). "The anatomy of anatomy: a review for its modernization" en Anatomical Sciences Education, 3(2), p. 83-93.

TOTH-COHEN, S. (1995). "Computer-assisted instruction as a learning resource for applied anatomy and kinesiology in the occupational therapy curriculum" en American Journal of Occupatonal Therapy, 48(8), p. 821-827. 